\title{
Resiliencia y disposición al pensamiento crítico en estudiantes de una universidad privada de Lima Metropolitana
}

\author{
Resilience and disposition towards critical thinking in students of a private \\ university in Metropolitan Lima
}

\author{
Liliana Cieza-Guevara ${ }^{1}$, Rocío Palomino-Ccoillo ${ }^{2}$ \\ Universidad Privada del Norte
}

Recibido: $05-05-20$

Aceptado: $12-06-20$

\begin{abstract}
Resumen
La presente investigación tuvo como finalidad determinar la relación entre la resiliencia y la disposición hacia el pensamiento crítico en estudiantes universitarios. El estudio correspondió al nivel descriptivo, de tipo sustantivo y con diseño correlacional. La muestra lo conformaron 301 ingresantes de diferentes facultades, de ambos sexos, entre 17 a 25 años de edad. En el recojo de datos se aplicó la Escala de Resiliencia (ER) de Wagnild y Young, versión adaptada por Novella en el 2002 y la Escala de disposición hacia el pensamiento crítico elaborado por Escurra y Delgado en el 2008 (quienes refieren que el instrumento fue construido bajo el modelo de Samejima a partir de la estructura conceptual desarrollada por Facione y Facione, que lo propusieron en 1995). Los resultados hallados confirmaron la hipótesis planteada, que existe una relación significativa entre la resiliencia y la disposición al pensamiento crítico en ingresantes de una universidad privada de Lima Metropolitana, al relacionar cada dimensión de resiliencia, se encontró correlación moderada entre las dimensiones de resiliencia: confianza en sí mismo, perseverancia y sentirse bien solo con la disposición al pensamiento crítico; mientras que la dimensión de ecuanimidad y satisfacción personal, lograron correlación baja. Otros resultados hallados fueron que los estudiantes alcanzaron niveles altos en ambas variables, y que no existen diferencias entre las dimensiones de resiliencia y de disposición al pensamiento crítico considerando el sexo y la carrera profesional.
\end{abstract}

Palabras clave: Resiliencia; disposición al pensamiento crítico; confianza en sí mismo; perseverancia y sentirse bien sólo.

\footnotetext{
${ }^{1}$ Docente Universidad Privada del Norte. Autor para correspondencia: E-mail: liliana.cieza@upn.edu.pe ORCID: https://orcid.org/0000-0002-6851-0432

${ }^{2}$ Docente Universidad Privada del Norte. E-mail: rocio.palomino@upn.edu.pe ORCID: https://orcid.org/0000-0001-9383-1759
}

(C) Los autores. Este artículo es publicado por la Revista de Investigación en Psicología de la Facultad de Psicología, Universidad Nacional Mayor de San Marcos. Este es un artículo de acceso abierto, distribuido bajo los términos de la licencia Creative Commons Atribucion - No Comercia_Compartir Igual 4.0 Internacional. (http://creativecommons.org/licenses/by-nc-sa/4.0/) que permite el uso no comercial, distribución y reproducción en cualquier medio, siempre que la obra original sea debidamente citada. 


\begin{abstract}
The purpose of this research was to determine the relationship between resilience and the disposition towards critical thinking in university students. The study corresponded to the descriptive level, of substantive type and with correlational design. The sample consisted of 301 students from different faculties, of both sexes, between 17 and 25 years of age. In the data collection, the Resilience Scale (ER) of Wagnild and Young was applied, a version adapted by Angelina Novella in 2002 and the Scale of readiness towards critical thinking elaborated by Escurra and Delgado (Instrument built under Samejima's model from the conceptual structure developed by Facione and Facione, 1995). The results confirmed the hypothesis, that there is a significant relationship between the resilience and the disposition to critical thinking in students from a private university in Metropolitan Lima, when relating each dimension of resilience, a moderate correlation was found between the dimensions of resilience: self-reliance, perseverance and existential aloneness with the disposition to critical thinking; while the dimension of equanimity and meaningfulness, achieved low correlation. Other results found were that students reached high levels in both variables, and that there are no differences between the dimensions of resilience and disposition to critical thinking considering sex and professional career.
\end{abstract}

Keywords: Resilience; disposition to critical thinking; self-reliance; perseverance; and existential aloneness.

El contexto global actual evidencia cambios económicos, sociales, de avance tecnológico y de mayor acceso al conocimiento, lo que nos permitiría deducir que las condiciones son favorables para el crecimiento y desarrollo de las poblaciones en general. Sin embargo, a nivel mundial la población de aproximadamente $1200 \mathrm{mi}$ llones jóvenes comprendidas entre las edades de 15 a 24 años (Fondo de las Naciones Unidas para la Infancia, 2011), se ven afectados significativamente en el campo laboral, y en acceso a espacios de salud y educativos de calidad, limitando su crecimiento personal y profesional. Dicha población juvenil en el Perú representa el "bono demográfico" que aportará en el futuro con la fuerza del trabajo para el crecimiento económico y sustentabilidad del país según el Informe Nacional de Juventudes elaborado por el Fondo de Población de las Naciones unidas (2015). Teniendo en cuenta este aspecto, el acceso a la educación superior de la población juvenil en nuestro país presenta una perspectiva preocupante, pues solamente el $27 \%$ de esta población logra acceder a este nivel de educación, representando un porcentaje bajo. En el caso de la educación universitaria los jóvenes que logran graduarse equivalen a un $6 \%$ de la población total que inicia estudios universitarios. (Instituto Nacional de Estadística [INEI], 2016).

Por otro lado, es importante precisar que la población de estudiantes que inician carrera universitaria se ubica dentro de la adolescencia tardía, etapa que tiene como características la consolidación de su personalidad, el planteamiento de metas de vida y el afrontamiento de diversas demandas (familiares, sociales, personales, académicas), contexto que difiere de sus etapas anteriores (Gonzales y Artuch, 2014). A su vez, es una población que es afectada por factores psicosociales, como 
la ansiedad y el estrés relacionados con el avance académico (Celis, Bustamante, Cabrera, Alarcón, y Monge, 2001).

De allí la importancia de conocer la realidad de la población de ingresantes universitarios, quienes deben adecuarse a dichas condiciones, desarrollando nuevas formas de pensamiento, actitudes y comportamientos, como parte del desarrollo de una personalidad resiliente que les permitan hacer frente a las adversidades y lograr metas para consolidar su proyecto de vida.

Revisando la literatura, se encuentra que la palabra resiliencia, etimológicamente deriva del latín resiliio, cuyo significado es saltar, rebotar, según Klotiarenco (1997 citado en Becoña, 2006). El término resiliencia como tal, tiene su origen en la física, entendido como característica de ciertos materiales para soportar altas presiones e impactos, y después recuperar su forma original (Barcelata, 2015).

Los investigadores Wagnild y Young (1993) consideran la resiliencia como la característica de la personalidad positiva que posee la persona y que favorece su adaptación a situaciones difíciles, como presiones u obstáculos.

Por otro lado Grotberg (2006), concibe la resiliencia como "la capacidad humana para, hacer frente a las adversidades de la vida, aprender de ellas, superarlas e inclusive, ser transformados por estas" (p. 18).

En relación al pensamiento crítico, según Ennis (1986 citado por Escurra y Delgado, 2008) es "el pensamiento racional, reflexivo, interesado en qué hacer o creer. De manera que es el tipo de pensamiento que se caracteriza por manejar y dominar las ideas" (p. 144). El pensamiento crítico tiene como funciones, el revisar, evaluar y repasar ideas, que han sido entendidas, procesadas y comunicadas mediante otros tipos de pensamiento como el verbal, matemático o lógico, no siendo su objetivo del pensamiento crítico, generar ideas, sino revisarlas. Otro aporte importante se da en 1990 con el Informe Delphi (citado por Rodríguez, 2011) documento que definió al pensamiento crítico como el proceso de autorregulación, que posee finalidad, y produce la interpretación, el análisis, la evaluación y la inferencia; mediante lo cual se puede explicar las evidencias en las que se basa dicho juicio. A partir de dicho documento, surge la propuesta de Facione en 1990, quien define al pensamiento crítico como la formación de un juicio deliberado y autorregulado que se usa para interpretar, analizar, evaluar, inferir y explicar las ideas, hechos, situaciones, experiencias, etc. (citado por Olivares y Heredia, 2012).

Así, Escurra y Delgado (2008) respecto al pensamiento crítico, afirman que dos elementos lo componen: las habilidades cognitivas y la disposición para ponerlo en práctica. Según Facione (2000 citado por Escurra y Delgado, 2008) define al segundo componente como el "conjunto de atributos caracterológicos personales para valorar y utilizar el pensamiento crítico, es decir que constituye la motivación hacia 
el uso de las habilidades del pensamiento crítico" (p.144), que son expresadas por actitudes y valores, constituyéndose en dimensiones de la personalidad.

Los antecedentes de investigación revelan que, en su mayoría, se han centrado en el constructo resiliencia y el abordaje del aspecto emocional, relegando la importancia de relacionarlo con el pensar crítico y la disposición a ponerlo en práctica frente a situaciones de conflicto o de toma de decisiones, siendo necesario conocer dicha relación, específicamente en ámbitos universitarios, donde se prioriza el pensamiento racional.

$\mathrm{Al}$ respecto la literatura internacional, nos refiere la relación existente entre resiliencia y rendimiento académico, encontrando en los estudios factores personales que permiten protegerse ante situaciones difíciles (Alonso-Aldana, Beltrán-Márquez, Máfara-Duarte y Gaytán-Martínez, 2016). Un estudio, de tipo correlacional en estudiantes universitarios evidenció una relación directa entre satisfacción positiva con la vida, la resiliencia y la reparación emocional, indicando la importancia de fomentar la resiliencia y la educación de la inteligencia emocional para mejorar el bienestar personal (Cejudo, López-Delgado y Rubio, 2016). Otros investigadores, encontraron que factores resilientes relacionados con introspección, iniciativa e independencia muestran niveles más elevados en estudiantes de cuarto año que sus pares más jóvenes (López, Martínez, Serey, Velasco y Zúñiga, 2013).

Dentro de la literatura peruana, se encuentran pocos estudios relacionados con los constructos resiliencia y pensamiento crítico, es así que Motta (2017), con su investigación evidenció que existe entre resiliencia, actitud creativa hacia actividades académicas y habilidad del pensamiento crítico, una relación directa, en una población de ingresantes a la universidad.

En referencia a los estudios de resiliencia, Peralta, Ramírez y Castaño (2006), en estudiantes con bajo y alto rendimiento académico, hallaron marcadas diferencias respecto en los factores resilientes; así mismo encontraron que existen menos factores resilientes en el grupo de menor rendimiento académico. En la misma línea, Caldera, Aceves y Reynoso (2016) hallaron altos niveles de resiliencia en los estudiantes universitarios, no evidenciando diferencias significativas considerando las variables carrera, sexo y edad. Respecto a investigaciones.

En relación con el pensamiento crítico, Villa (2012), halló relación entre la inteligencia emocional y la motivación para el pensamiento crítico, en estudiantes de licenciatura; pero no, entre la inteligencia emocional y el rendimiento académico; similar resultado encontró Rodríguez (2016), cuyos resultados demostraron que existe relación entre el pensamiento crítico y la autoestima en la población de ingresantes universitarios.

Por lo mencionado anteriormente, el presente estudio se formula con el objetivo principal de determinar relación entre la resiliencia y la disposición 
hacia el pensamiento crítico en ingresantes de una universidad privada de Lima Metropolitana. Así como, relacionar las dimensiones de la resiliencia con la disposición al pensamiento crítico de la población estudiada.

\section{MÉTODO}

\section{Diseño}

La investigación es de tipo cuantitativo, de diseño descriptivo correlacional, en la medida que pretende determinar el grado de relación existente entre dos variables en una muestra de sujetos, para lo cual se utiliza el coeficiente de correlación como análisis estadístico (Sánchez y Reyes, 2015).

\section{Población}

En la investigación realizada, la población la conformaron 5300 estudiantes ingresantes de diversas carreras profesionales del semestre 2018-1 de una universidad particular de Lima Metropolitana, que respetando lo establecido por las normativas éticas que regulan el ejercicio profesional (Código de Ética del Colegio de Psicólogos del Perú), se procuró la protección de la confidencialidad de la información personal e institucional.

\section{Muestra}

Han participado como muestra final 301 ingresantes debido que se registró inconsistencia en los protocolos aplicados. El tamaño muestral fue de ambos sexos, con edades que fluctuaban entre 17 a 25 años y de diversas carreras; utilizando un muestreo no probabilístico de tipo accidental, debido a que los participantes llevaban un curso con una u otra investigadora. La muestra fue determinada con la fórmula propuesta por Abad y Servin, 1981; Hernández, 2010 (citados por Grajeda, 2018).

$$
\begin{gathered}
\text { NZ } 2 \text { p }(1-p) \\
\text { n=-1N-1)e } 2+Z 2 \text { p } 1 \text {-p) } \\
5300(1.96) 20.5(1-0.5) \\
n=358
\end{gathered}
$$

Donde:

$\mathrm{N}=$ Población de estudio 5300

$\mathrm{p}=$ Eventos favorables 0.5 
$\mathrm{Z}=$ Nivel de significación 1.96

$\mathrm{e}=$ Margen de error 0.05

\section{Variables de estudio}

\section{Definición conceptual}

Resiliencia, según Wagnild y Young (1993) es la característica de una personalidad positiva que favorece la adaptación individual a situaciones difíciles, como presiones u obstáculos; mientras que la disposición al pensamiento crítico, es conceptuada como el conjunto de atributos caracterológicos personales para valorar y utilizar el pensamiento crítico, por lo que representa la motivación para ejecutar las habilidades del pensamiento crítico. Facione y Facione (2000 citado por Escurra y Delgado, 2008).

\section{Definición operacional}

Las variables de estudio corresponden a los indicadores de la Escala de Resiliencia (ER) de Wagnild y Young (1993), compuesta por cinco dimensiones: Ecuanimidad, perseverancia, confianza en uno mismo, satisfacción personal y sentirse bien sólo. Y la Escala de Disposición hacia el Pensamiento Crítico de Escurra y Delgado (2008), compuesta de siete dimensiones: Capacidad de análisis, búsqueda de la verdad, ser sistemático, confiar en el razonamiento, curiosidad, madurez para formular juicios y la amplitud mental.

\section{Técnicas e instrumentos de recolección de datos}

La recolección de datos se realizó luego del consentimiento informado de los estudiantes, en aplicaciones colectivas de los instrumentos.

\section{Instrumentos}

Escala de Resiliencia, siendo sus autores Wagnild Gail M. y Young Heather M.; adaptada en el Perú por Novella (2002). Escala que evalúa 2 factores: Competencia personal (17 ítems) y Aceptación de uno mismo y de la vida (8 ítems); expresados en 5 dimensiones: ecuanimidad, perseverancia, confianza en uno mismo, satisfacción personal y sentirse bien solo.

\section{Validez y confiabilidad de la prueba original}

Wagnild y Young, aplicaron la validez de contenido de modo a priori, obteniendo una validez concurrente, ya que correlacionaron la resiliencia con: depresión, con $\mathrm{r}=-0.36$, satisfacción de vida, $\mathrm{r}=0.59$; moral, $\mathrm{r}=0.50$; autoestima, $\mathrm{r}=0.57$; y percepción al estrés, $\mathrm{r}=-0.67$. En 1993, Wagnild y Young, hallaron una confiabilidad de 0.85 en una muestra de cuidadores de enfermos de Alzheimer; luego en dos muestras de estudiantes mujeres ya graduadas resultó 0.86 y 0.85 ; en madres primerizas 
post parto 0.90; Al evaluar la confiabilidad por el método de la consistencia interna mediante del Alpha de Cronbach, hallando para toda la escala, un valor de 0.94. En el Perú Novella (2002), logró adaptar la Escala de Resiliencia original en una prueba piloto y obtuvo por el método de la consistencia interna con el alfa de Cronbach, una confiabilidad de 0.88 .

\section{Validez y confiabilidad de la prueba adaptada}

En el presente estudio, desde un enfoque intrapruebas, se determinó el coeficiente de correlación entre las dimensiones y el puntaje total de la escala. Respecto a la correlación se utilizó el coeficiente Rho de Spearman (los puntajes tienen una distribución no paramétrica); los resultados, indican correlaciones positivas y estadísticamente muy significativas $(\mathrm{p}<0,001)$ entre todas las dimensiones y el puntaje total de la Escala de Resiliencia; siendo los coeficientes indicadores de correlaciones que oscilan entre niveles moderados a altos (Delgado, Escurra y Torres, 2006). Estos datos apoyan la unidimensionalidad de la prueba, por lo que es sostenible que las cinco dimensiones de la escala tienen validez factorial.

Se analizó la confiabilidad de escala global, mediante el método de consistencia interna, se obtuvo un coeficiente alfa de Cronbach final de 0,852 , por ello se señala que el instrumento es confiable; sin embargo, al analizar el índice de homogeneidad, también conocido como correlación ítem-test corregido (IH) y el coeficiente de confiabilidad alfa de Cronbach por cada una de las dimensiones de la Escala de Resiliencia, se eliminaron los ítems cuyos índices de homogeneidad eran menores a 0,20 , quedando de esta forma constituida la escala por 23 ítems, eliminándose los ítems 20 y 25 .

Escala de disposición hacia el pensamiento crítico, elaborada por Escurra y Delgado (2008) quienes refieren que el instrumento fue construido con el modelo de Samejima a partir de la estructura conceptual desarrollada por Facione y Facione, que lo propusieron en 1995, evalúa siete dimensiones: Capacidad de análisis, búsqueda de la verdad, ser sistemático, confiar en el razonamiento, curiosidad, madurez para formular juicios y la amplitud mental.

\section{Validez y confiabilidad de la prueba original}

Escurra y Delgado validaron la escala mediante criterio de jueces en la validez de contenido y mediante el modelo de respuesta graduada de Samejima se procedió al análisis de cada ítem, para la validez de constructo bajo la teoría clásica de los tests. En cuanto a la confiabilidad, los autores, aplicaron el análisis de confiabilidad generalizada por consistencia interna mediante el coeficiente alfa de Cronbach, llegando a concluir que las correlaciones ítem-test revisadas se ubican entre 0.72 (Curiosidad) y 0.93 (Capacidad de análisis). También se halla un valor de 0.96 en el coeficiente de confiabilidad, indicando que es significativo $(\mathrm{p}<.05)$, 
además el cálculo de los intervalos de confianza al 99\% refieren que se ubica de 0.95 a 0.97 la verdadera confiabilidad.

\section{Validez y confiabilidad de la prueba adaptada}

Se determinó la validez de la escala adaptada, mediante el análisis factorial exploratorio para cada dimensión, teniendo como criterio mínimo para pertenecer a un factor una saturación mínima de 0,30 (saturación = correlación del ítem con el factor), encontrándose que los ítems alcanzan saturaciones o cargas factoriales cercanos o superiores a 0,50 , pudiendo señalarse que las dimensiones están factorialmente bien definidas. En relación con el enfoque intrapruebas, se determinó el coeficiente de correlación entre las dimensiones y la puntuación total de la escala. En la correlación se utilizó el coeficiente Rho de Spearman, observándose correlaciones positivas y estadísticamente muy significativos $(\mathrm{p}<0,001)$ entre todas las dimensiones y el puntaje total de la Escala de Disposición al Pensamiento Crítico; siendo los coeficientes indicadores de correlaciones que oscilan entre niveles moderados a altos. El coeficiente de confiabilidad para la escala global fue igual a 0,933 , permitiendo señalar que el instrumento es confiable. Así también se eliminaron aquellos ítems cuyos índices de homogeneidad eran negativos o menores a 0,20 , quedando constituida la escala final por 61 ítems. Los ítems eliminados fueron los siguientes: 1, 6, 7, 14, 20, 27, 48, 57 y 64 .

Respecto a cada una de las dimensiones del test, se encuentran que las dimensiones confianza en la razón $(0,791)$, capacidad de análisis $(0,727)$ y ser sistemático $(0,708)$ alcanzan los coeficientes más elevados; seguidos por las dimensiones madurez para formular juicios $(0,684)$, amplitud mental $(0,661)$ y curiosidad $(0,628)$; la dimensión que obtiene el coeficiente más bajo es la dimensión búsqueda de la verdad $(0,527)$. Como se observa los coeficientes alcanzan valores mínimos aceptables. Estos datos, coeficientes de confiabilidad e índices de homogeneidad permiten señalar que el instrumento es confiable.

\section{RESULTADOS}

\section{Resultados estadísticos descriptivos}

De manera general los resultados revelan los niveles de resiliencia de la muestra total, observándose que, en todas las dimensiones, más del 90\% de los estudiantes ingresantes a una universidad de Lima Metropolitana, logran alcanzar la categoría alto y muy alto de resiliencia, el resto del porcentaje se ubica en un nivel medio, solo el $0,7 \%$ se ubica en un nivel bajo específicamente en la dimensión ecuanimidad. Estos datos permiten señalar que la muestra evaluada alcanza puntuaciones elevadas en cuanto a resiliencia. 
Tabla 1

Niveles de Resiliencia según muestra total

\begin{tabular}{lccccc}
\hline \multirow{2}{*}{ Dimensiones } & \multicolumn{5}{c}{ Niveles } \\
\cline { 2 - 6 } & $\begin{array}{c}\text { Muy bajo } \\
\mathbf{f}(\mathbf{\%})\end{array}$ & $\begin{array}{c}\text { Bajo } \\
\mathbf{f}(\mathbf{\%})\end{array}$ & $\begin{array}{c}\text { Medio } \\
\mathbf{f ( \% )}\end{array}$ & $\begin{array}{c}\text { Alto } \\
\mathbf{f}(\mathbf{\%})\end{array}$ & $\begin{array}{c}\text { Muy alto } \\
\mathbf{f}(\mathbf{\%})\end{array}$ \\
\hline Confianza en sí mismo & 0 & 0 & $7(2,3)$ & $25(8,3)$ & $269(89,4)$ \\
Ecuanimidad & 0 & $2(0,7)$ & $24(8)$ & $96(31,9)$ & $179(59,5)$ \\
Perseverancia & 0 & 0 & $3(1)$ & $58(19,3)$ & $240(79,7)$ \\
Satisfacción personal & 0 & 0 & $11(3,7)$ & $46(15,3)$ & $244(81,1)$ \\
Sentirse bien solo & 0 & 0 & $10(3,3)$ & $22(7,3)$ & $269(89,4)$ \\
\hline Resiliencia & 0 & 0 & $3(1)$ & $31(10,3)$ & $267(88,7)$ \\
\hline
\end{tabular}

De otro lado, en referencia a la variable disposición al pensamiento crítico en la muestra total, se observa una tendencia hacia los niveles alto y muy alto (ver tabla 2). Respecto a cada dimensión, se encontró que en la dimensión búsqueda de la verdad, se observa que el $56,4 \%$ se ubica en un nivel alto y muy alto, y el $18,9 \%$ en un nivel bajo a muy bajo; en la dimensión amplitud mental, el 38,2\% en un nivel de alto a muy alto, y el $26,6 \%$ en niveles bajos; en la dimensión capacidad de análisis, el 48,2\% en niveles altos, y el 23,2\% en niveles bajos; en la dimensión ser sistemático el $43,2 \%$ en niveles altos y el 33,3\% en niveles bajos; en la dimensión confianza en la razón, el 41,1\% en niveles altos, y el 30,9\% en niveles bajos; en la dimensión curiosidad, el 44,2\% en niveles altos, y el 36,2\% en niveles bajos; y finalmente en la dimensión madurez para formular juicios, el $44,8 \%$ en niveles altos y el $24,6 \%$ en niveles bajos. Es decir, aproximadamente 4 de cada 10 evaluados alcanzan niveles altos en cuanto a disposición al pensamiento crítico, 3 de cada 10 aproximadamente alcanzan un nivel medio, y 2 o 3 de cada 10 evaluados se ubican en niveles bajos.

Tabla 2

Niveles de Disposición al Pensamiento Crítico según muestra total

\begin{tabular}{lccccc}
\hline \multicolumn{1}{c}{ Dimensiones } & \multicolumn{5}{c}{ Niveles } \\
\cline { 2 - 6 } & $\begin{array}{c}\text { Muy bajo } \\
\mathbf{f ( \% )}\end{array}$ & $\begin{array}{c}\text { Bajo } \\
\mathbf{f ( \% )}\end{array}$ & $\begin{array}{c}\text { Medio } \\
\mathbf{f ( \% )}\end{array}$ & $\begin{array}{c}\text { Alto } \\
\mathbf{f}(\%)\end{array}$ & $\begin{array}{c}\text { Muy alto } \\
\mathbf{f}(\%)\end{array}$ \\
\hline Búsqueda de la verdad & $20(6,6)$ & $37(12,3)$ & $74(24,6)$ & $110(36,5)$ & $60(19,9)$ \\
Amplitud mental & $42(14)$ & $38(12,6)$ & $106(35,2)$ & $69(22,9)$ & $46(15,3)$ \\
Capacidad de análisis & $19(6,3)$ & $51(16,9)$ & $86(28,6)$ & $67(22,3)$ & $78(25,9)$ \\
Ser sistemático & $33(11)$ & $67(22,3)$ & $71(23,6)$ & $74(24,6)$ & $56(18,6)$ \\
Confianza en la razón & $33(11)$ & $60(19,9)$ & $84(27,9)$ & $48(15,9)$ & $76(25,2)$ \\
Curiosidad & $41(13,6)$ & $68(22,6)$ & $59(19,6)$ & $99(32,9)$ & $34(11,3)$ \\
Madurez para formular juicios & $36(12)$ & $38(12,6)$ & $92(30,6)$ & $78(25,9)$ & $57(18,9)$ \\
\hline Pensamiento crítico & $15(5)$ & $61(20,3)$ & $74(24,6)$ & $85(28,2)$ & $(21,9)$ \\
\hline
\end{tabular}




\section{Resultados de la contrastación de las hipótesis}

Para conocer los estadísticos más apropiados para el análisis y las contrastación de las hipótesis, se realizó un análisis de normalidad de la distribución de las puntuaciones de las variables, para ello se aplicó el coeficiente Kolmogorov Smirnov con la corrección de la significación de Lilliefors, siendo estos valores significativos $(\mathrm{p}<0,01)$ y muy significativos $(\mathrm{p}<0,001)$ en todas las variables, por lo que se determinó realizar las correlaciones y comparaciones con estadísticos no paramétricos; por lo que se utilizó el coeficiente Rho de Spearman, y para las comparaciones el coeficiente U de Mann-Whitney (para dos grupos de contraste) y el coeficiente Kruskal-Wallis (para más de dos grupos de contraste).

Como se observa en la Tabla 3, existe una correlación moderada y positiva de 0,593 , y estadísticamente significativa en relación con las variables de estudio resiliencia y la disposición hacia el pensamiento crítico. Lo cual indica que a mayor resiliencia mayor es la disposición hacia el pensar crítico.

Tabla 3

Coeficiente de correlación de Spearman entre resiliencia y disposición al pensamiento crítico

\begin{tabular}{lcc}
\hline & \multicolumn{2}{c}{ Resiliencia } \\
\cline { 2 - 3 } & Rs & $\mathbf{P}$ \\
\hline Disposición al pensamiento critico & $0,593 * * *$ & 0,000 \\
\hline $\mathrm{N}=301, * * * \mathrm{p}<0,001$ &
\end{tabular}

Respecto a la primera hipótesis especifica (H1), que plantea: "Existe relación significativa entre la dimensión de resiliencia: confianza en sí mismo con la disposición al pensamiento crítico en ingresantes de una universidad privada de Lima Metropolitana", se ha encontrado que existe una correlación estadísticamente muy significativa $(\mathrm{p}<0,001)$ y positiva $(\mathrm{rs}=0,558)$ entre ambas, y siendo la fuerza de correlación moderada (Delgado, Escurra y Torres, 2006) por ello se confirma dicha hipótesis específica; en cuanto a la segunda hipótesis específica (H2) "Existe relación significativa entre la dimensión de resiliencia: ecuanimidad con la disposición al pensamiento crítico en ingresantes de una universidad privada de Lima Metropolitana", se halla una correlación estadísticamente muy significativa $(\mathrm{p}<0,001)$ y positiva $(\mathrm{rs}=0,361)$ y el coeficiente es indicador de una correlación baja entre ambas (Delgado, Escurra y Torres, 2006); ante ello se confirma la hipótesis en referencia; respecto a la tercera hipótesis específica (H3) "Existe relación significativa entre la dimensión de resiliencia: perseverancia con la disposición al pensamiento crítico en ingresantes de una universidad privada de Lima Metropolitana", se encuentra una relación muy significativa ( $\mathrm{p}$ $<0,001)$ y positiva $(\mathrm{rs}=0,548)$, siendo el coeficiente indicador de una correlación moderada (Delgado, Escurra y Torres, 2006), aceptándose por tanto la hipótesis 
en referencia; mientras que para la hipótesis específica (H4) "Existe relación significativa entre la dimensión de resiliencia: satisfacción personal con la disposición al pensamiento crítico en ingresantes de una universidad privada de Lima Metropolitana", se halla un valor estadísticamente muy significativo $(\mathrm{p}<0,001)$ y positivo ( $\mathrm{rs}=0,217)$, con un coeficiente de correlación baja entre ambas (Delgado, Escurra y Torres, 2006); por lo que se acepta dicha hipótesis específica; y respecto a la quinta hipótesis específica (H5) "Existe relación significativa entre la dimensión de resiliencia: sentirse bien solo con la disposición al pensamiento crítico en ingresantes de una universidad privada de Lima Metropolitana" que establece una relación entre ambas, se halla un valor muy significativo $(\mathrm{p}<0,001)$, y el coeficiente de correlación de Spearman resulta positivo $(r s=0,439)$, siendo indicador de una correlación moderada (Delgado, Escurra y Torres, 2006). Estos datos permiten aceptar la hipótesis específica mencionada.

\section{Resultados complementarios}

Entre los resultados obtenidos, también se halló que no existen diferencias en cuanto a sexo; respecto a las dimensiones de resiliencia entre varones y mujeres, se han encontrado valores no significativos $(p>0,05)$ en casi todas las dimensiones, a excepción de la dimensión sentirse bien solo, en la cual se halla diferencias estadísticamente significativas ( $p<0,05)$, siendo los varones aquellos que obtienen un rango promedio mayor, es decir, en cuanto a la dimensión sentirse bien solo, los varones obtienen una puntuación mayor; y respecto a las dimensiones de disposición al pensamiento crítico, los datos expresan valores no significativos $(\mathrm{p}>0,05)$ en todos los casos.

Otro resultado encontrado fue que no existen diferencias entre las dimensiones de resiliencia y de disposición al pensamiento crítico considerando la carrera profesional. Así respecto a las dimensiones de resiliencia en cuanto a la carrera profesional, no se hallan diferencias estadísticamente significativas $(p>0,05)$ en casi todas las dimensiones a excepción de la dimensión ecuanimidad $(\mathrm{p}<0,01)$; similar resultado se halló con valores no significativos ( $\mathrm{p}>0,05)$, al estudiar la disposición del pensamiento crítico desde sus dimensiones según la carrera profesional en curso.

\section{DISCUSIÓN}

Los datos encontrados en esta investigación evidencian la correlación entre resiliencia y disposición hacia el pensamiento crítico, variables estudiadas en ingresantes a la universidad; institución que tiene como uno de sus fines el preparar profesionales que la sociedad requiere a corto y largo plazo, así como el producir y actualizar conocimientos, tal como refiere Chuaqui (2012), la universidad implica la preparación de la persona, le permite formar su identidad, en la que cabe la reflexión introspectiva, donde el estudiante se construye y reconstruye 
de forma continua, por lo que la universidad no solo es formativa, sino también transformativa para el estudiante, buscando que la persona trascienda mediante la criticidad, innovación y creatividad, en un marco de libertad e individualidad, y es en estas condiciones donde surge la persona resiliente; en específico cuando debe afrontar situaciones desconocidas, nuevas y/o problemáticas, que implican riesgo; así se motiva para reflexionar y se dispone a hacer uso de su pensamiento crítico, iniciando este proceso al observar, fijar su atención en los puntos relevantes, en re-observar desde diferentes perspectivas su realidad, analiza el problema y le otorga una resignificación, extrae conclusiones, planifica acciones, se establece objetivos a lograr y toma decisiones. A todo este proceso es fundamental que el estudiante mantenga el control de las emociones (autoregulación), asumiendo firmemente que el pensamiento racional le permitirá analizar, criticar, evaluar y asumir decisiones para lograr adaptarse positivamente a la realidad y por ende ser transformado.

Los resultados hallados en el presente estudio, se respaldan en investigaciones realizadas por Motta (2017) que encuentra relación directa con relación a las variables resiliencia, habilidades del pensamiento crítico y creatividad en estudiantes universitarios, evidenciando que ante situaciones adversas, los estudiantes, mantuvieron el equilibrio emocional y demostraron superior nivel en la habilidad del pensamiento crítico para diversos temas; y en la de Obando (2018), que halló una relación positiva entre la resiliencia académica, la meta de aproximación al aprendizaje y la teoría de desarrollo de la inteligencia. También tenemos a Quiñones (2007), quién refiere que el individuo es agente de su propia transformación y adaptación social; así mismo Martin (2013) refiere que el resiliente es una persona reflexiva, que analiza la situación, de modo que le permita abordarla inteligentemente identificando sus aspectos positivos y negativos, para enfocarse de manera constructiva en el afrontamiento. Así mismo, Wolin y Wolin (1999) y Melillo y Suarez (2002) afirman que el perfil del resiliente se caracteriza por preguntarse y generar una respuesta a sí mismo, caracterizado por examinar y analizar sus ideas y argumentos, realizar autoexamen y autocorrección (se motiva a utilizar las destrezas y habilidades del pensamiento crítico).

Analizando la relación hallada, entre la dimensión de resiliencia: confianza en sí mismo con la disposición al pensamiento crítico en ingresantes; referimos que el individuo resiliente, es aquel que cree en sí mismo, que tiene la certeza de poseer habilidades y destrezas, que le permiten enfrentar y adaptarse a las dificultades universitarias; así Grotberg (2006), señala que la persona resiliente no solo aprende a confiar en los demás, sino en ellos mismos. Melillo, Suarez y Rodriguez (2004), consideran como base de la resiliencia la autoestima consistente; del mismo modo, Rodriguez (2011) agrega que la persona desarrolla su integridad, confianza, autonomía, empatía, entereza y humildad, al identificar la razón como herramienta en la toma de decisiones. Así también la Organización 
Panamericana de la Salud (1998), señala que entre los atributos de la persona resiliente se encuentra la alta autoestima (concepción positiva de sí mismo), control de las emociones y de los impulsos, la autonomía y el sentido del humor.

Se encontró en los ingresantes una relación entre la dimensión ecuanimidad de la variable resiliencia y la disposición al pensamiento crítico, esto implica que están en proceso de lograr una percepción equilibrada de sus vivencias, de asumir con serenidad las situaciones adversas propias del contexto universitario; esto es respaldado por la Organización Panamericana de la Salud (1998), que afirma que diversos estudios han demostrado que el control de las emociones y de los impulsos tienen una asociación positiva con la posibilidad de ser resiliente; de igual modo, Quiñones (2007) menciona que una característica de la persona resiliente es la percepción de control que posee de una situación, lo que le permitirá afrontarla.

Respecto a la relación entre la dimensión de resiliencia: perseverancia con la disposición al pensamiento crítico en ingresantes; se interpreta que presentan voluntad para mantenerse y persistir hacia el logro de sus objetivos; haciendo uso de la reflexión, del análisis, de resignificar su realidad y a partir de allí construir y ejecutar respuestas innovadoras. Asi, Aquino (2016) y Villa (2012) encontraron características resilientes con capacidad de poder ajustarse rápidamente a sucesos difíciles en contextos universitarios. Mientras que Cyrulnik (2004 citado por Rodriguez, Morell y Fresneda, 2015), afirma que las personas resilientes utilizan las vivencias adversas para aprender y crecer, ya que cuentan con una gran tenacidad y motivación intrínseca que les ayudan a mantenerse constantes y seguras de sí mismas; así como la Organización Panamericana de la Salud (1998), identifica como factores predictivos de resiliencia, el poseer una dirección hacia objetivos y la consecución de los mismos (tener éxito en lo que se emprenda), motivación para los logros, fe en un futuro mejor, sentido para anticiparse y coherencia.

En el análisis de la relación hallada entre la dimensión de resiliencia: satisfacción personal con la disposición al pensamiento crítico en ingresantes; así el estudiante asume a la universidad como medio para alcanzar metas trazadas y trascender, por lo que requiere otorgar y construir un sentido de vida coherente, que guíe sus acciones y decisiones personales y profesionales, esto obliga al individuo resiliente a tener la disposición de analizar su realidad ordenando sus ideas y pensar críticamente, parafraseando a Maganto y Maganto (2013).

Referente a la relación encontrada entre sentirse bien solo con la disposición al pensamiento crítico en ingresantes, se debe incidir que la resiliencia en la persona se da en libertad; en esa libertad es que puede alcanzar realizar su yo, siendo lo que realmente es. Así el sentirse bien estando solo, le genera intimidad consigo mismo, encontrando un espacio y momento que le permita desarrollar su capacidad 
creadora, disponerse a pensar, a analizar, a comprender lo que sucede, a ordenar sus ideas, siendo este "estar solo" el momento propicio para la introspección, de análisis y producto de ello, el estudiante se reconoce con capacidad de hacer frente a sus adversidades; esto lo corrobora la investigadora Grotberg (2006), cuando afirma que la persona resiliente aprende a ver la soledad como una oportunidad de crecimiento y desarrollo personal; que utiliza su inteligencia para decidir qué lineamientos y de qué modo los aplicará como expresión de la libertad personal. También señala que la autonomía es fundamental para promover factores resilientes y reforzar aquellos que ya se han activado.

\section{CONCLUSIONES}

- Al término del estudio realizado se puede concluir que los estudiantes más resilientes presentan mayor disposición al pensamiento crítico.

- De las dimensiones de resiliencia estudiadas en relación con la disposición al pensamiento crítico, se evidencia que, los estudiantes con mayor confianza en sí mismos, muestran mayor autoeficacia y confianza en sus decisiones con un mejor nivel en el pensar crítico frente a su realidad.

- Dentro de la variable resiliencia, en el análisis de las dimensiones los estudiantes con mayor ecuanimidad presentan características de equilibrio, autocontrol, con disposición de hacer mejor uso del pensar crítico.

- Los estudiantes que presentan mejores niveles en la dimensión perseverancia de la variable resiliencia, se caracterizan por asumir un rol activo para alcanzar sus objetivos, revelando una mayor disposición de su pensar crítico frente a situaciones de adversidad.

- En la variable resiliencia, la dimensión satisfacción personal, los estudiantes con mayores niveles otorgan un sentido coherente a su vida, comprendiendo y organizando lo que sucede a su alrededor presentando en este sentido una mayor disposición al pensamiento crítico.

- En la dimensión de la resiliencia: sentirse bien solo, los estudiantes con mejores niveles logran una adecuada intimidad consigo mismos evidenciándose de igual manera, una adecuada disposición al pesar crítico, que le permite adaptarse y transformarse frente a su realidad.

- Los estudiantes alcanzan niveles altos en ambas variables, además no se encontraron diferencias significativas entre sexo, ni considerando la carrera profesional; así los estudiantes, sin distingo de sexo y/o carrera profesional, presentan una adecuada adaptabilidad a situaciones adversas, motivados en el uso de sus habilidades para el pensar crítico. 


\section{Agradecimientos / Acknowledgments:}

Las autoras agradecen al Dr. Mario Bulnes Bedón, por el apoyo constante en la asesoría para esta investigación, a la Dra. Carmen Leni Álvarez Taco por las recomendaciones brindadas en el planteamiento del problema y a Mg. Marcos Paico Ruiz por el apoyo en el análisis estadístico de la investigación.

\section{Fuentes de financiamiento / Funding:}

La presente investigación y artículo han sido autofinanciados.

\section{Rol de los autores / Authors Roles:}

LC y RP: Realizaron la administración de las pruebas, redacción, procesamiento de datos, análisis estadístico, interpretación de resultados y corrección.

\section{Aspectos éticos / legales; Ethics / legals:}

En el presente estudio se ha respetado lo establecido por las normativas éticas que regulan el ejercicio profesional (Código de Ética del Colegio de Psicólogos del Perú). Se procuró la protección de la confidencialidad de la información personal e institucional, asegurando el anonimato de las personas e instituciones involucradas en la muestra.

\section{Conflicto de intereses / Competing interests:}

Ambas autoras declaran bajo juramento no haber incurrido en conflicto de interés al realizar este artículo.

\section{REFERENCIAS}

Alonso-Aldana, R., Beltrán-Márquez, Y., Máfara-Duarte, R., y Gaytán-Martínez, Z. (2016). Relación entre rendimiento académico y resiliencia en una universidad tecnológica. Revista de Investigaciones Sociales, 2(4), 38-49.

Aquino, J. (2016). Adaptación a la vida universitaria y resiliencia en becarios (Tesis de pregrado). Pontificia Universidad Católica del Perú, Lima. Recuperado de http://tesis.pucp.edu.pe/repositorio/handle/123456789/7706

Barcelata, B. (2015). Adolescentes en riesgo. Una mirada a partir de la relisiencia. México: Manual Moderno.

Becoña, E. (2006). Resiliencia: Definición, características y utilidad del concepto. Revista de Psicopatología y Psicología Clinica, 11(3), 125-146. doi:https://doi.org/10.5944/ rppc.vol.11.num.3.2006.4024

Caldera, J., Aceves, B., y Reynoso, O. (2016). Resiliencia en estudiantes universitarios. Un estudio comparado entre carreras. Psicogente, 19(36), 227-239. doi:http://dx.doi. org $/ 10.17081 /$ psico.19.36.1294 
Cejudo, J., López-Delgado, M., y Rubio, M. (2016). Inteligencia emocional y resiliencia: su influencia en la satisfacción con la vida en estudiantes universitarios. Anuario de Psicología, 42(2), 51-57. doi: http://dx.doi.org/10.15381/anales.v62i1.4143

Celis, J., Bustamante, M., Cabrera, D., Cabrera, M., Alarcón, W., y Monje, E. (2001). Ansiedad y Estrés Académico en Estudiantes de Medicina Humana del Primer y Sexto Año. Anales de la Facultad de Medicina, 62(1), 25-30.

Chuaqui, T. (2012). El rol de la universidad no es solo formativa, sino también transformativa. Psicología, educación y universidad. XIX coloquio de estudiantes de psicología de la Pontificia Universidad Católica del Perú. Lima, Perú. Recuperado de: https:// puntoedu.pucp.edu.pe/entrevistas/rol-universidad-no-solo-formativa-tambien-transformativa/

Delgado, A. Escurra, L. y Torres, W. (2006). La medición en psicología y educación: teorías y aplicaciones. Lima: Hozlo.

Escurra, L., y Delgado, A. (2008). Relación entre disposición hacia el pensamiento crítico y estilos de pensamiento en alumnos universitarios de Lima Metropolitana. Persona, (11), 143-175.

Fondo de las Naciones Unidas para la Infancia. (2011). Estado mundial de la Infancia 2011. Recuperado de https://www.unicef.org/bolivia/UNICEF_-_Estado_Mundial de_la_Infancia_2011__La_adolescencia_una_epoca_de_oportunidades.pdf

Fondo de Población de las Naciones Unidas. (2015). Informe Nacional de las Juventudes en el Perú-2015. Recuperado de http://www.unfpa.org.pe/publicaciones/publicacionesperu/SENAJU-Informe-Nacional-Juventudes-2015.pdf

Gonzales, M., y Artuch, R. (2014). Perfiles de resiliencia y estrategias de afrontamiento en la universidad: Variables contextuales y demográficas. Electronic Journal of Research in Educational Psychology, 12(3), 621-648. doi: http://dx.doi.org/10.14204/ ejrep.34.14032

Grajeda, A. (2018). Construcción y validación de un test de aptitud funcional selectora visual en estudiantes de escuelas estatales del primer grado de primaria del distrito de Jesús María, 2018 (Tesis de Doctorado). Universidad César Vallejo, Perú. http://repositorio.ucv.edu.pe/bitstream/handle/UCV/21249/Grajeda_MAT. pdf? sequence $=1 \&$ isAllowed $=\mathrm{y}$

Grotberg, E. (2006). La resiliencia en el mundo de hoy. Como superar las advesidades. España: Gedisa.

Instituto Nacional de Estadística e Informática. (2016). Pobreza y gasto social. Recuperado de https://www.inei.gob.pe/estadisticas/indice-tematico/sociales/

López, Y., Martínez, C., Serey, Y., Velasco, A, y Zúñiga, C. (2013). Factores de resiliencia en estudiantes de carreras pedagógicas de una universidad estatal de la Región de Valparaíso. Revista de Psicología Universidad Viña del Mar. 3(5), 76-89.

Maganto, C. y Maganto, J. (2013). Cómo potenciar las emociones positivas y afrontar las negativas. Madrid: Pirámide.

Martín, L. (2013). La personalidad resiliente. Madrid: Síntesis. 
Melillo, A. y Suárez, E. (2002). Descubriendo las propias fortalezas. Argentina: Paidós.

Melillo, A., Suárez, E. y Rodríguez, D. (2004). Resiliencia y subjetividad. Los ciclos de la vida. Argentina: Paidós.

Motta, N. (2017). Resiliencia, actitud creativa hacia las actividades académicas y habilidad del pensamiento crítico de los estudiantes del primer ciclo de la Escuela Académico Profesional de Educación de la Facultad de Educación de la Universidad Nacional Mayor de San Marcos (Tesis de maestría). Universidad Nacional Mayor de San Marcos, Perú.

Novella, A. (2002). Incremento de la resiliencia luego de la aplicación de un programa de psicoterapia breve en madres adolescentes (Tesis de maestría). Universidad Nacional Mayor de San Marcos, Perú.

Obando, K. (2018). Resiliencia académica, metas de logro, teorías implícitas y alto rendimiento (Tesis de maestría). Pontificia Universidad Católica del Perú.

Olivares, S., y Heredia, Y. (2012). Desarrollo del pensamiento crítico en ambientes de aprendizaje basado en problemas en estudiantes de Educación. Revista mexicana de investigación educativa, 13(54), 759-778.

Organización Panamericana de la Salud. (1998). Manual de identificación y promoción de la resiliencia en niños y adolescentes. Recuperaddo de http://www1.paho.org/hq/ dmdocuments/2009/Resilman.pdf

Peralta, S., Ramírez, A. y Castaño, H. (2006). Factores resilientes asociados al rendimiento académico en estudiantes pertenecientes a la Universidad de Sucre. Revista Psicología desde El Caribe, 17(1), 196-219.

Quiñones, M. (2007) Resiliencia: Resignificación creativa de la adversidad. Bogotá: Universidad Distrital de Francisco José de Caldas.

Rodríguez, M. (2011). Pensamiento crítico y aprendizaje. Mexico: Limusa

Rodriguez, M., Morell, J., y Fresneda, J. (2015). Manual de promoción de la

resliciencia infantil y adolescente. Madrid: Pirámide.

Rodríguez, D. (2016). Pensamiento crítico y autoestima en ingresantes de la Universidad Nacional de Ingeniería 2016 (Tesis de Maestría). Universidad César Vallejo, Perú.

Sanchez, H. y Reyes, C. (2015). Metodología y diseños en la investigación cientifíca. Lima: Business Support Aneth.

Villa, N. (2012). Inteligencia emocional, motivación para el pensamiento crítico y rendimiento académico en estudiantes de psicología (Tesis de Maestría). Universidad Autónoma de Nuevo León, México.

Wagnild, G. \& Young, H. (1993). Development and psychometric evaluation of the Resilience Scale. Journal of Nursing Measurement, 1(2), 165-178.

Wolin, S. \&Wolin, S. (1999). Morality in COAs: Revisiting the Syndrome of Over-Responsibility. Recuperado de http://projectresilience.com/article12.htm 
